Para citar este artículo: Palenzuela Zanca, J., Marcos Ramos, M., \& González de Garay, B. (2021). Análisis del discurso sobre la diversidad funcional en los personajes de las series televisivas españolas. Anuario Electrónico de Estudios en Comunicación Social "Disertaciones", 14(2), 1-17. https://doi.org/10.12804/revistas.urosario.edu.co/disertaciones/a.10121

\title{
ANÁLISIS DEL DISCURSO SOBRE LA DIVERSIDAD FUNCIONAL EN LOS PERSONAJES DE LAS SERIES TELEVISIVAS ESPAÑOLAS
}

Discourse Analysis of Functional Diversity in Spanish TV Series' Characters

Análise do discurso sobre diversidade funcional nos caracteres da série de televisão espanhola

Julia Palenzuela Zanca, Universidad de Salamanca (España)

julia.palenzuelaz@usal.es

María Marcos Ramos, Universidad de Salamanca (España)

julia.palenzuelaz@usal.es

Beatriz González de Garay, Universidad de Salamanca (España)

julia.palenzuelaz@usal.es

Recibido: 14 de enero de 2021

Aprobado: 21 de enero de 2021

Fecha de prepublicación: 14 de mayo de 2021 


\title{
DISERTACIONES
}

AVANCES

\section{RESUMEN}

En el presente artículo se analizan los resultados obtenidos en un estudio previo sobre la representación de la diversidad funcional en las series de producción españolas emitidas en horario de prime time en las seis cadenas generalistas españolas durante la segunda mitad del año 2016, el año 2017 y la primera mitad del año 2018. Este análisis mostraba que exclusivamente el $1,2 \%$ de los personajes poseían algún tipo de diversidad funcional, además de no ocupar en ninguno de los casos papeles protagónicos. Con los datos previos, se procedió al análisis discursivo de las intervenciones de estos personajes, fijando los objetivos en el estudio de la autonomía mostrada, la persecución de objetivos narrativos y la relevancia de estos personajes en la historia, haciendo una crítica desde la perspectiva del modelo de vida independiente. Las conclusiones obtenidas a raíz del análisis de la muestra de quince capítulos de ficción apoyan la postura de que no solamente existe una carencia cuantitativa de personajes con diversidad funcional en la ficción española, sino que también se da una insuficiencia de su relevancia narrativa y una ausencia del modelo de vida independiente en ella.

Palabras clave: diversidad funcional; series; prime time; análisis de discurso; representación.

\begin{abstract}
This article assesses the results obtained from a prior study concerning the representation of functional diversity across Spanish-produced TV series broadcasted in prime time by six Spanish general-interest channels during the second semester of 2016, both semesters of 2017, and the first semester of 2018. The results indicated that only $1,2 \%$ of the fictional characters showed signs of any type of functional diversity; none of these characters had leading roles. Previous data were used to perform a discourse analysis of these characters' participation, with the objective of studying their autonomy, the pursuit of the narrative goal, and the significance of said characters in the stories through an independent living model-based review. The conclusions drawn from the analysis of a sample comprising 15 episodes support the view that evinces not only the limited number of characters with functional diversity in Spanish TV fiction but also the poor narrative significance of such characters as well as the absence of a consideration of the independent living model.
\end{abstract}

Keywords: Functional diversity; series; prime time; discourse analysis; representation.

\section{RESUMO}

Este artigo analisa os resultados obtidos em um estudo anterior sobre a representação da diversidade funcional em séries de produção espanholas veiculadas em horário nobre nos seis canais generalistas espanhóis durante o segundo semestre de 2016, 2017 e primeiro semestre de 2018. Essa análise mostrou que apenas 1,2\% dos personagens apresentavam algum tipo de diversidade funcional, além de não ocuparem protagonistas em nenhum dos casos. Com os dados anteriores, procedeu-se à análise funcional das intervenções desses personagens, 


\section{DISERTACIONES}

AVANCES

Comunicación y controversias sociocientíficas en salud y medio ambiente

ISSN: $1856-9536$

Doi: https://doi.org/10.12804/revistas.urosario.edu.co/disertaciones/a.10121

Volumen 14, Número 2 / Julio-diciembre 2021

Versión PDF para imprimir desde

http://revistas.urosario.edu.co/index.php/disertaciones

estabelecendo os objetivos no estudo da autonomia manifestada, a prossecução dos objetivos narrativos e a relevância destes personagens na história, fazendo uma crítica na perspectiva do Modelo de Vida Independente. As conclusões obtidas a partir da análise da amostra de 15 capítulos de ficção corroboram a posição de que não só há uma carência funcional de personagens com diversidade funcional na ficção espanhola, mas também há uma insuficiência de sua relevância funcional e uma ausência do Modelo de Vida Independente nele.

Palavras-chave: diversidade funcional; séries; horário nobre; análise do discurso; representação.

\section{Introducción}

En la actual sociedad de la información, donde la exposición a mensajes audiovisuales es constante y cada vez mayor (Miranda, 2003; Tabernero et al., 2009), el análisis del consumo cada vez es más relevante. El objetivo de este estudio es analizar la manera en el que la diversidad funcional es representada en la ficción española. Para ello, en un primer lugar, se recurrió a un análisis de contenido cuantitativo cuya muestra de personajes con diversidad funcional no resultó lo suficientemente amplia como para poder realizar operaciones estadísticas significativas, ya que había claramente una infrarrepresentación muy acusada, lo que es, sin duda, significativo de cómo es la ficción audiovisual nacional. En concreto, los resultados obtenidos indicaban que exclusivamente un 1,2\% ( $n=$ 15) de la muestra correspondía a personajes con diversidad funcional. Es decir, de los 1237 personajes analizados, exclusivamente 15 manifestaban algún tipo de diversidad funcional (Palenzuela et al., 2019). En el momento en que se obtuvieron los resultados, se detectó la necesidad de completar el análisis de contenido con un estudio de corte cualitativo de los casos en los que estos personajes aparecían en la ficción y así poder ultimar la radiografía del modelo de representación nacional de la diversidad funcional.

Además, este artículo pretende complementar el análisis de los personajes con diversidad funcional en la ficción española con las bases del modelo de vida independiente (Palacios \& Romañach, 2006), que sostiene que los funcionamientos biológicos pueden reflejar distintos estados físicos, psíquicos o sensoriales. Sin embargo, las capacidades de las personas no se ven afectadas por los distintos modelos de funcionamiento ante las diferentes realidades que se plantean a diario, sino que las capacidades son el modo en el que las personas se enfrentan a distintas situaciones. Por lo tanto, es un modelo que se aleja de la concepción de que las personas con diversidad funcional automáticamente están relacionadas con tener una enfermedad, una minusvalía o una discapacidad, sino que aboga por que las personas, según sus capacidades, se enfrentarán a situaciones de manera diversa, lo que no significa que haya una manera más correcta que otra de solventar una situación concreta (Mejía, 2020; Palacios \& Romañach, 2006, 2008; Romañach \& Lobato, 2009). 


\section{DISERTACIONES}

AVANCES

\section{Estado de la cuestión}

El retrato audiovisual de determinados colectivos y grupos sociales ha evolucionado históricamente y el colectivo de personas con diversidad funcional no es una excepción. La representación de la discapacidad ha ido variando a lo largo de los años, evolucionado desde ser considerada como un castigo divino hasta la figura de la persona valiente que se sobrepone a las malas experiencias vitales, representadas por la capacidad del sujeto de ver la belleza de la vida a pesar del "impedimento" de la diversidad funcional (Areces, 2017). Además, la idea mayoritaria actual con relación a la representación de la diversidad funcional está basada en la visión de que esta es un obstáculo que hace que la persona no pueda realizar sus objetivos y que viva enfadada por ello o bien que necesite el apoyo de una persona sin diversidad funcional para poder llegar a alcanzar aquello que se ha propuesto. Este último es el caso de la película Campeones (Javier Fesser, 2018), donde los personajes con diversidad funcional son un apoyo para que el protagonista, completamente normativo, cumpla sus objetivos narrativos y personales en la historia. Los personajes con diversidad funcional en esta película son una excusa, unos sujetos infantilizados que, a pesar de mostrar autonomía en sus actividades diarias, son representados de manera dependiente de los personajes normativos. Debido a este tipo de visiones, en cuanto a la representación de grupos minoritarios en la ficción y bajo la premisa de que la comunicación es mucho más efectiva y tiene mucho más calado en el espectador cuando se utiliza un código que apela a las emociones y a los sentimientos (Ferrés, 2003; Ferrés \& Masanet, 2017; Peñamarín, 2001; Ugalde et al., 2012), se vuelve necesario la realización de artículos que analicen la vía en la que son representados estos grupos. Esto se ve potenciado si se tiene en cuenta que los medios de comunicación de masas tienen una gran responsabilidad a la hora de establecer discursos, ya que esos mensajes que van a ser consumidos y procesados por una audiencia pueden llegar a influir o, más bien, a perpetuar determinadas opiniones acerca de la realidad social (Igartua \& Humanes, 2004), dado que intervienen de manera directa en su modelación ideológica (Van Dijk, 2005). Son una base fundamental para entender el modo en el que observamos el mundo y la manera en la que nos relacionamos con él (Belmonte \& Guillamón, 2008; Del Río \& Álvarez, 2004; Fajardo, 2006; Herrero \& Tovar, 2011; Peñamarín, 2001). Por ello, la necesidad de la educación en el consumo audiovisual es primordial para la formación de sujetos críticos (Belmonte \& Guillamón, 2008; Galán, 2006), lo que es especialmente importante cuando se trata de niños y de jóvenes, puesto que su consumo es mayor y está mucho más diversificado que el de los adultos (Belmonte \& Guillamón, 2008; Del Río \& Álvarez, 2004; Peñamarín, 2001).

Así mismo, la elección del análisis de contenido de ficción estaría justificado, además de por la importancia que tiene a nivel de transmisión ideológica, por el aumento y cambios sufridos en su modelo de uso. Actualmente, la presencia de internet en los hogares españoles es del 91,4\% (Instituto Nacional de Estadística, 2019), lo que significa que la mayor parte de hogares españoles tienen acceso a la mayor biblioteca audiovisual del mundo y que pueden acceder a ella desde distintos tipos de dispositivos y en un horario adaptado. Los hábitos de consumo de contenidos audiovisuales, por lo tanto, se han visto modificados, ya que la familia ya no se reúne delante de la televisión para ver todo el mismo programa, sino que cada miembro de la familia puede acceder a un contenido personalizado (Miranda, 2003). Siguiendo con esta idea, el control del consumo audiovisual cada vez es más relevante, por lo que la alfabetización mediática comienza a ser una necesidad social, pues el acceso a internet por parte de la 


\section{DISERTACIONES}

AVANCES

Comunicación y controversias sociocientificas en salud y medio ambiente

ISSN: 1856-9536

Doi: https://doi.org/10.12804/revistas.urosario.edu.co/disertaciones/a.1012.1

Volumen 14, Número 2 / Julio-diciembre 2021

Versión PDF para imprimir desde

http://revistas.urosario.edu.co/index.php/disertaciones

población es mayoritario y, por lo tanto, el acceso a la información ilimitado (Del Río \& Álvarez, 2004). Para ello, es fundamental la ruptura con la construcción de identidades mediante estereotipos, dado que estos simplifican la realidad y ayudan a identificar lo que es normal y lo que no (Belmonte \& Guillamón, 2008). Sin embargo, si asumimos que la realidad social está construida, aceptamos el hecho de que la normatividad, y lo que está fuera de ella, también es un producto social que dependería y variaría en función de la cultura en la que nos situamos (Lacalle \& Gómez, 2018; Vázquez, 2008). Por ello, la diversidad funcional, y el propio término que esta implica, es una ruptura con la normalidad previa (Moscoso, 2015; Toboso, 2013; Vázquez, 2008), ya que toda persona que funcione de manera 'diferente' a lo que previamente se ha establecido como normativo estaría dentro del grupo de personas con diversidad funcional. Por lo tanto, sería la sociedad y no la manera en la que se llevan a cabo las acciones ni la enfermedad o síndrome asociada a esta lo que conllevaría la exclusión (Mejía, 2020; Moscoso, 2015; Palacios \& Romañach, 2006, 2008; Romañach \& Lobato, 2009; Toboso, 2013; Vázquez, 2008). Por ejemplo, el uso de un aparato que apoye carencias auditivas en las personas mayores no significaría una diversidad funcional, puesto que el uso de estos en esta franja de edad es considerado como 'normal'. Sin embargo, si el mismo aparato lo usa un niño, automáticamente asociamos que ese niño tiene una 'discapacidad' (Palacios \& Romañach, 2006).

Es por esta lógica por la que se ha decidido usar el término de hombres y mujeres con diversidad funcional, el cual aparece por primera vez en el año 2005 en el Foro de Vida Independiente, donde se rechazan las palabras usadas hasta ese momento, como minusvalía o discapacidad (Romañach \& Lobato, 2009). Además, uno de los ejes fundamentales en los que se apoya este estudio es desarrollar las directrices del modelo de vida independiente, basadas en que la incapacidad para realizar determinadas acciones no anula el hecho de que la persona con diversidad funcional pueda decidir sobre estas, dado que el mismo hecho de concebir lo que es normal y lo que no estaría basado en un constructo social referente a la normalidad (Palacios \& Romañach, 2006; Vázquez, 2008). En este texto, se plantea que la visión por parte de la sociedad hacia el colectivo de las personas con diversidad funcional ha variado a lo largo de la historia. Para ello, se sigue la catalogación que realizan Agustina Palacios y Javier Romañach (2006) de varios modelos, de los cuales en los tres primeros no existe una diferenciación entre diversidad funcional y discapacidad. Esto cambia cuando se alcanza el cuarto modelo conocido como el modelo de vida independiente, el primero que hace una separación entre enfermedad y diversidad funcional, y que a la vez reivindica este último término, el de hombres y mujeres con diversidad funcional, como la expresión más correcta, puesto que implica una consideración de este colectivo como independiente y autónomo, reclamando, a su vez, la perspectiva de una sociedad diversa que no presupone unos preceptos de normatividad (Mejía, 2020; Palacios \& Romañach, 2006).

Sin embargo, antes de llegar al modelo de vida independiente, se propone que la visión social de la diversidad funcional ha pasado por varios modelos. El primero se corresponde al modelo de prescindencia, caracterizado por una percepción de la diversidad funcional como parte de un castigo divino que penaliza a la persona que 'sufre' esta anomalía con una existencia dura y sin esperanzas. Seguido de este modelo y debido a las dos guerras mundiales acontecidas en el siglo xx, la visión de la diversidad funcional (o discapacidad, ya que en ese momento estaban unidas) evoluciona hacia el conocido como modelo rehabilitador, que considera la diversidad funcional como una enfermedad a la que hay que poner cura. Sin embargo, esta perspectiva cambió cuando se alcanzó el modelo social, el cual propone que no son los hombres y mujeres con diversidad funcional los que han de superar ninguna enfermedad o adaptarse a la sociedad, sino que es la sociedad la que es necesario que supere la presunción de la 


\section{DISERTACIONES}

AVANCES

normalidad. Es decir, si la sociedad elimina dicho supuesto, no sería necesario que ningún sujeto cambiase para encajar en los preceptos sociales previos (Palacios \& Romañach, 2006).

Por otro lado, para el acercamiento de la industria audiovisual española actual a un modelo de representación acorde a las directrices del modelo de vida independiente, se propone como primer paso un análisis del modelo audiovisual español en relación con la representación de personas con diversidad funcional y así poder plantear alternativas más inclusivas, que promuevan la autonomía de las personas con diversidad funcional y que esté alejado de los estereotipos narrativos actuales.

\section{Objetivos}

Los objetivos marcados en esta investigación vienen determinados por textos previos que analizan la situación de este colectivo dentro de la sociedad española. Teniendo en cuenta la ausencia de documentos oficiales que se refieran al término diversidad funcional, se ha recurrido a aquellos que usan palabras como discapacidad o minusvalía para establecer la bibliografía base. Así, el "Informe Olivenza 2017, sobre la situación general de la discapacidad en España” (Jiménez \& Huete, 2017), la “Estrategia Española sobre Discapacidad 2012-2020" (Real Patronato sobre Discapacidad, 2011) o el Decreto Legislativo 1/2013, del 29 de noviembre (BOE, 2013) sirven de base para este análisis, ya que de lo que se trata es de analizar la situación de este colectivo en el contexto de ficción de producción nacional. Igualmente, debido a que se ha apostado por el término diversidad funcional, se decidió recurrir al escrito "El modelo de la diversidad: la bioética y los derechos humanos como herramientas para alcanzar la plena dignidad en la diversidad funcional" (Palacios \& Romañach, 2006) como herramienta guía para poder basar en un texto validado la crítica hacia el modelo de representación actual, lo que nos permite realizar una crítica pautada y sustentada.

Este artículo pretende aprovechar los mecanismos de socialización de los medios de comunicación de masas para poder crear sociedades más igualitarias e inclusivas. Los objetivos marcados se basarían en el análisis de dos ejes fundamentales. Por una parte, la autonomía del personaje basada en la independencia, que a su vez estaría definida por la capacidad de este para poder alcanzar sus objetivos narrativos planteados durante la narración. Y, por otro lado, se trata de analizar la autonomía de las personas con diversidad funcional como sujeto y la capacidad de relacionarse sin que haya una intervención de otras personas.

Se han propuesto cuatro preguntas de investigación que dotan una dirección más concreta hacia la que dirigir el análisis planteado. Al no existir un corpus de literatura científica previa suficiente que centrase el análisis en las series de ficción y en el papel que la diversidad funcional tiene en ellas, se rechazó la idea de proponer hipótesis.

1. ¿Qué tipo de representación se sigue en los discursos de ficción nacional para abordar la diversidad funcional?

2. ¿Qué tipo de relaciones, tanto en el contexto sexo-afectivo como en el de amistad, se plantea para las personas con diversidad funcional en los capítulos recogidos en la muestra?

3. ¿Se puede establecer que el modelo de vida independiente es la visión predominante en el modo de representar a las personas con diversidad funcional en la ficción española?

4. ¿Existe una alusión directa a la diversidad funcional manifestada en la narración por parte de los personajes que intervienen en ella? 


\section{DISERTACIONES}

AVANCES

Comunicación y controversias sociocientíficas en salud y medio ambiente

ISSN: $1856-9536$

Doi: https://doi.org/10.12804/revistas.urosario.edu.co/disertaciones/a.10121

Volumen 14, Número 2 / Julio-diciembre 2021

Versión PDF para imprimir desde

http://revistas.urosario.edu.co/index.php/disertaciones

\section{Metodología}

El método seguido para la realización de este estudio es un análisis cualitativo de los resultados obtenidos en el artículo "Representación de la diversidad funcional en series contemporáneas españolas de prime time" (Palenzuela et al., 2019), mediante el cual se analizaron 1237 personajes de ficción, repartidos en 47 capítulos emitidos en las seis cadenas generalistas españolas: La 1, La 2, Antena 3, Cuatro, Telecinco y La Sexta durante la segunda mitad del año 2016, el año 2017 completo y la primera mitad del año 2018. Esta investigación tuvo como resultado que solamente quince personajes de toda la muestra mostraban en la narración algún signo visible de diversidad funcional, lo que significaba tan solo un 1,2\% de toda la muestra recogida. Esto ha supuesto que, al ser los resultados poco representativos a nivel estadístico, fuese necesario un análisis más personalizado a cada uno de los personajes ilustrados en la muestra, analizando situaciones concretas, el grado de autonomía mostrado, el objetivo narrativo de cada uno de ellos y las relaciones que se establecen con el resto de los personajes.

En la tabla 1 se presentan los capítulos de las series en los que aparecen los personajes con diversidad funcional, aunque solo se han podido incluir los nombres de los actores que representan a los personajes con diversidad funcional con mayor peso narrativo.

Tabla 1. Descripción de la muestra

\begin{tabular}{|c|c|c|c|c|}
\hline Serie & Capítulo & $\begin{array}{l}\text { Rol del } \\
\text { personaje }\end{array}$ & $\begin{array}{l}\text { Nombre del } \\
\text { actor }\end{array}$ & $\begin{array}{l}\text { Tipo de diversidad } \\
\text { funcional representada }\end{array}$ \\
\hline $\begin{array}{l}\text { El chiringuito de Pepe } \\
\text { (Telecinco, 2014-2016) }\end{array}$ & $\begin{array}{c}\text { S2e01 } \\
\text { "Tradición" }\end{array}$ & Secundario & $\begin{array}{l}\text { Juan Manuel } \\
\text { Montilla }\end{array}$ & Física \\
\hline $\begin{array}{l}\text { Mar de plástico } \\
\text { (Antena 3, 2015-2016) }\end{array}$ & $\begin{array}{c}\mathrm{S} 02 \mathrm{e} 13 \\
\text { "La última palabra" }\end{array}$ & Secundario & $\begin{array}{l}\text { Federico } \\
\text { Aguado }\end{array}$ & Mental \\
\hline $\begin{array}{l}\text { El Ministerio del Tiempo } \\
\text { (La 1, 2015-2016) }\end{array}$ & $\begin{array}{c}\text { S02e01 } \\
\text { "Tiempo de leyenda" }\end{array}$ & Background & * & Física \\
\hline $\begin{array}{l}\text { Cuéntame cómo pasó } \\
\text { (TVE, 2001-actualidad) }\end{array}$ & $\begin{array}{c}\text { S17e03 } \\
\text { "Lo que aprendí" }\end{array}$ & Background & * & Física \\
\hline $\begin{array}{l}\text { Víctor Ros } \\
(\text { La 1, 2015-16) }\end{array}$ & $\begin{array}{c}\text { S02e02 } \\
\text { "Centauros de Sierra } \\
\text { Morena" }\end{array}$ & Background & * & Física \\
\hline $\begin{array}{l}\text { El incidente } \\
\text { (Antena 3, 2017) }\end{array}$ & $\begin{array}{c}\text { S01E01 } \\
\text { "En el ojo del huracán" }\end{array}$ & Secundario & Diego Martín & Física y mental \\
\hline $\begin{array}{l}\text { El incidente } \\
\text { (Antena 3, 2017) }\end{array}$ & $\begin{array}{l}\text { S01E01 } \\
\text { "En el ojo del huracán" }\end{array}$ & Secundario & $\begin{array}{l}\text { Celso Bugallo } \\
\text { Aguiar }\end{array}$ & Mental \\
\hline $\begin{array}{l}\text { El incidente } \\
\text { (Antena 3, 2017) }\end{array}$ & $\begin{array}{c}\text { S01E01 } \\
\text { "En el ojo del huracán" }\end{array}$ & Background & * & Física \\
\hline $\begin{array}{l}\text { Ella es tu padre } \\
\text { (Telecinco, 2017-2018) }\end{array}$ & $\begin{array}{c}\text { S01E01 } \\
\text { "Just like a woman" }\end{array}$ & Secundario & Aníbal Gómez & Física \\
\hline
\end{tabular}




\section{DISERTACIONES}

AVANCES

Comunicación y controversias sociocientificas en salud y medio ambiente

\begin{tabular}{|c|c|c|c|c|}
\hline Serie & Capítulo & $\begin{array}{l}\text { Rol del } \\
\text { personaje }\end{array}$ & $\begin{array}{c}\text { Nombre del } \\
\text { actor }\end{array}$ & $\begin{array}{c}\text { Tipo de diversidad } \\
\text { funcional representada }\end{array}$ \\
\hline $\begin{array}{l}\text { Cuéntame cómo pasó } \\
\text { (TVE, 2001-actualidad) }\end{array}$ & S019E02 "Silencio y plomo" & Secundario & $\begin{array}{l}\text { Miguel } \\
\text { Canalejo }\end{array}$ & Mental \\
\hline $\begin{array}{l}\text { La verdad } \\
\text { (Telecinco, 2018) }\end{array}$ & $\begin{array}{c}\text { S01E01 } \\
\text { "La jaula abierta..." }\end{array}$ & Secundario & Paco Marín & Mental \\
\hline $\begin{array}{l}\text { Cuerpo de Élite } \\
\text { (Antena 3, 2018) }\end{array}$ & $\begin{array}{l}\text { S01E01 } \\
\text { "El sobrino del rey" }\end{array}$ & Secundario & $\begin{array}{l}\text { Juan Manuel } \\
\text { Montilla }\end{array}$ & Física \\
\hline $\begin{array}{l}\text { El accidente } \\
\text { (Telecinco, 2017-2018) }\end{array}$ & $\begin{array}{c}\text { S01E01 } \\
\text { "Adiós, amor" }\end{array}$ & Secundario & $\begin{array}{l}\text { Eusebio } \\
\text { Poncela }\end{array}$ & Física \\
\hline $\begin{array}{l}\text { Fugitiva } \\
\text { (TVE, 2018) }\end{array}$ & $\begin{array}{l}\text { S01E01 } \\
\text { "La salida" }\end{array}$ & Secundario & Iván Pellicer & Física \\
\hline $\begin{array}{l}\text { El continental } \\
\text { (TVE, 2018) }\end{array}$ & $\begin{array}{c}\text { S01E01 } \\
\text { "O pagas o cobras" }\end{array}$ & Secundario & $\begin{array}{l}\text { Christian } \\
\text { Sánchez }\end{array}$ & Mental \\
\hline
\end{tabular}

Nota: solo se han incluido en la tabla aquellos personajes con un mayor peso narrativo.

Fuente: elaboración de las autoras.

Después de realizar el análisis estadístico correspondiente y debido a que el valor de $p$ es $<0,05$, se puede afirmar que es estadísticamente significativo (Igartua, 2006) el hecho de que ninguno de los personajes representados en la muestra tenga un papel protagonista. Por otro lado, en papeles secundarios el porcentaje es del $66,7 \%$ y en papeles de background aparecía un $33,3 \%$ de personajes con diversidad funcional $\left(X^{2}[2, n=1232]=8071\right.$, $p<0,018$ ) (tabla 2).

Tabla 2. Tipo de personaje

\begin{tabular}{l|cc|c}
\multirow{2}{*}{ Tipo de personaje } & \multirow{2}{*}{$\%$ total } & \multicolumn{2}{c}{ Rasgo de diversidad funcional } \\
\cline { 3 - 5 } & & No posee diversidad funcional & Posee diversidad funcional \\
\hline Protagonista & 11,7 & 11,8 & 0,0 \\
\hline Secundario & 33,4 & $33-$ & $66,7+$ \\
\hline Background & 54,9 & 55,1 & 33,3 \\
\hline
\end{tabular}

Nota: - Valor estadísticamente inferior al porcentaje total (análisis estándar de residuos corregidos); + Valor estadísticamente superior al porcentaje total (análisis estándar de residuos corregidos); $\left(X^{2}[2, n=1232]=8071, p<0,018\right)$.

Fuente: recuperado de Palenzuela et al. (2019). 


\section{DISERTACIONES}

AVANCES

Comunicación y controversias sociocientificas en salud y medio ambiente

ISSN: 1856-9536

Doi: https://doi.org/10.12804/revistas.urosario.edu.co/disertaciones/a.10121

Volumen 14, Número 2 / Julio-diciembre 2021

Versión PDF para imprimir desde

http://revistas.urosario.edu.co/index.php/disertaciones

Al quedar validado que los resultados son estadísticamente significativos, se puede afirmar que tiene relevancia el hecho de que ningún personaje ocupe un papel protagonista. Sin embargo, al querer hacer un análisis acerca de los personajes que ocupan un papel secundario o de background es cuando se ha evidenciado que la muestra era demasiado pequeña como para poder llegar a conclusiones mediante la estadística exclusivamente, por lo que es necesario analizar de forma cualitativa cómo son y cómo están estos personajes representados en la ficción televisiva nacional.

El análisis que se ha propuesto para contestar a las preguntas planteadas ha seguido varios pasos. En primer lugar, como ya se analizó a nivel cuantitativo la presencia de estos en la muestra, se ha intentado no fijar la atención en los personajes normativos, sino en las interacciones que los personajes con diversidad funcional tenían en la narración. Por un lado, se ha buscado que los personajes con diversidad funcional tuviesen un objetivo narrativo que alcanzar; por otro, el modelo de representación de la diversidad funcional que aparece en pantalla. Además, para que las respuestas a las preguntas de investigación planteadas sean consistentes, se ha de seguir un método comparativo validado. Para ello, se ha tenido en cuenta la literatura científica previa que ha marcado las pautas para poder establecer un análisis ordenado de la situación de los personajes con diversidad funcional en la narrativa española. Como consecuencia de no haber obtenido resultados estadísticamente representativos en el análisis anterior, ya que la presencia de estos personajes se limita a papeles secundarios $(66,7 \% ; n=11)$ o de background $(33,3 \% ; n=4)$ con ausencia total de personajes en papeles principales en la narración, se ha decidido recurrir a una distinción de estos en los diferentes modelos propuestos por el texto "El modelo de la diversidad. La bioética y los derechos humanos como herramientas para alcanzar la plena dignidad en las personas con diversidad funcional" (Palacios \& Romañach, 2006), con el objetivo de poder analizar cada una de las casuísticas de manera más individualizada.

\section{Discusión}

Si tomamos como referencia el texto "El modelo de la diversidad. La bioética y los derechos humanos como herramientas para alcanzar la plena dignidad en las personas con diversidad funcional” (Palacios \& Romañach, 2006) y la definición que aportan del modelo de prescindencia, del modelo rehabilitador y del modelo social, se puede hablar de que existen tres grupos en la muestra, en cuanto a representación de personajes con diversidad funcional: la representación desde la perspectiva de la enfermedad, la representación desde el encuadre del personaje como un sujeto malvado y, por último, la representación del personaje como un sujeto bueno, amable y al que moralmente poco se le puede recriminar, lo que coincidiría con las bases de los modelos anteriormente indicados.

En relación con la muestra seleccionada, el primer grupo relacionado con el modelo de prescindencia, donde los personajes aparecen como sujetos con un comportamiento reprochable, estaría nutrido, en primer lugar, por Mar de plástico (Antena 3, 2015-2016), donde el personaje con diversidad funcional que aparece es Sergio, hijo de uno de los hombres más ricos de la zona, Juan Rueda. Sergio se muestra como un personaje completamente dependiente de sus cuidadores, sobre todo de su hermano Fernando, por el cual es manipulado para que lleve a cabo una serie de actos delictivos, incluyendo el asesinato de su propio padre. 


\section{DISERTACIONES}

AVANCES

Comunicación y controversias sociocientificas en salud y medio ambiente

ISSN: 1856-9536

Doi: https://doi.org/10.12804/revistas.urosario.edu.co/disertaciones/a.10121

Volumen 14, Número 2 / Julio-diciembre 2021

Versión PDF para imprimir desde

http://revistas.urosario.edu.co/index.php/disertaciones

La justificación que se aplica dentro de la narración a la relación tan dependiente de los dos hermanos es que mientras que el padre de Sergio le esconde y le humilla, su hermano le trata bien y le cuida. Sin embargo, lo que percibimos durante todo el capítulo es un discurso paternalista, que infantiliza a Sergio y donde es necesario un trato de cuidado, ya que podría estallar en una crisis de locura en cualquier momento.

RODolfo: Alguien te ayudó a ser un asesino.

SERGIO: No, lo he hecho yo solo.

RODOLFO: ¿Estás seguro? Piénsalo, has hecho cosas muy complicadas.

SERGIO: Yo sé hacer cosas complicadas, yo sé hacer cosas complicadas.

Rodolfo: Vale, Sergio, vale. Vamos a hablar de tu hermano.

El segundo caso sería el de la serie La verdad (Telecinco, 2018), donde el personaje con diversidad funcional es el secuestrador que vende a Paula, personaje principal de la narración, momento que esta aprovecha poder escapar. La diversidad funcional que este manifiesta es psíquica, a raíz de la cual desarrolla una personalidad malvada con muy pocos escrúpulos. Además, se le representa como un pervertido sexual que secuestra chicas para prostituirlas. Por otro lado, no se nombra ni se hace referencia ninguna a su diversidad funcional. Se evidencia mediante la narración que el personaje no es normativo, pero al mismo tiempo solo se resalta el hecho de que es malvado por la diversidad funcional que posee.

MADRE: Digan lo que digan, no era un mal chico. Tenía eso, pero no era un mal chico.

El siguiente caso que se ha incluido en este grupo es el de Víctor Ros (TVE, 2015-2016), donde el personaje aparecido ocupa un papel de background conocido como 'El Manco', un bandido que guarda una moneda de oro dentro de la prótesis de su mano y que, a la vez, es la pista para que el protagonista solucione el caso. Aparece retratado como un bandolero alcohólico, que es asesinado en una tasca mientras se emborrachaba.

Además del caso nombrado, el caso de la serie El accidente (Telecinco, 2017-2018) es curioso, dado que el personaje con diversidad funcional que aparece en el capítulo analizado es Joao, un misterioso hombre que está en silla de ruedas y que se enfada al descubrir a Lucía, personaje principal del capítulo, en una rueda de prensa. Es empresario y un importante cliente para la empresa de camiones que pertenece a José (marido de Lucía) y Juan, hermano de José. Además, se presenta como un hombre con mucho carácter y agresivo, aparte de evidenciar que su fuente de ingresos y su negocio no es legal.

JOAO: Espero que no muevan la mercancía que me han robado por mi propio territorio.

Como último capítulo por analizar dentro del grupo del modelo de la prescindencia se trata de la serie Cuerpo de Élite (Antena 3, 2018), donde el personaje con diversidad funcional que aparece es Carreño, representado por Juan Manuel Montilla. Este es un parlamentario de ideología progresista y que presenta objetivos narrativos con relación a acabar con el Cuerpo de Élite, grupo policial secreto que está nutrido por los personajes principales de la serie. Es un personaje que tiene ambiciones, que persigue un objetivo narrativo y personal claro y que se muestra con autonomía en la toma de decisiones que afectan directamente a la consecución de sus objetivos, los cuales lo señalan como el antihéroe de la narración. En el capítulo analizado se hace referencia a su diversidad funcional cuando es el propio personaje quien la realiza. En concreto, cuando se hace referencia a las barreras arquitectónicas que impiden que Carreño se desplace de manera autónoma.

CARREÑO: Vaya ministerio lleno de trabas y escalones. 


\section{DISERTACIONES}

AVANCES

Comunicación y controversias sociocientificas en salud y medio ambiente

ISSN: 1856-9536

Doi: https://doi.org/10.12804/revistas.urosario.edu.co/disertaciones/a.10121

Volumen 14, Número 2 / Julio-diciembre 2021

Versión PDF para imprimir desde

http://revistas.urosario.edu.co/index.php/disertaciones

En el siguiente modelo, el rehabilitador, están incluidas cuatro series: Cuéntame cómo pasó (TVE, 2001-actualidad), en la que el personaje con diversidad funcional es Abraham, hijo de la dueña del bar del barrio y que, gracias a él, el vecindario se entera del disparo recibido por María, hija pequeña de los Alcántara, por parte de la Policía en el transcurso de una manifestación estudiantil. Su aparición es breve y no cambia su presencia en el relato, pues la aportación que tiene en este es prescindible, solo transmite el mensaje, no lo cambia ni aporta nada a nivel discursivo, ni de sus propios intereses o voluntades como sujeto. El incidente (Antena 3,2017), donde pese a que los personajes con diversidad funcional son de background el tratamiento de estos corresponde a personajes enfermos; El Ministerio del Tiempo (TVE, 2015-2020), en el que la presencia de la diversidad funcional es meramente anecdótica, debido a que se trata de un personaje también de background que acude a la consulta médica que ocupa uno de los personajes protagonistas, y, por último, Fugitiva (TVE, 2018), donde el personaje con diversidad funcional es Rubén, hijo sordo de la protagonista Magda y el cual hace uso de un audífono, pese al rechazo que le supone tener que utilizarlo. Durante el capítulo, se puede observar una sobreprotección por parte de Magda hacia su hijo por la sordera. Por otro lado, también se introduce la idea de que por el hecho de pertenecer a una clase social más alta se tiene acceso a una serie de ventajas. Sin embargo, estas ventajas, que podrían dotar al personaje de una mayor autonomía, son anuladas por la sobreprotección de su madre.

MAGDA: Cuándo te vas a dar cuenta de que Rubén no es como los demás y que tiene sus propias limitaciones.

Para finalizar con el apartado de resultados obtenidos en el análisis, se presenta el último grupo de representación de personajes con diversidad funcional enmarcados en la catalogación nombrada. En este caso, se trata del modelo social, donde se hace hincapié en el hecho de que es la sociedad quien tiene el problema con relación a la diversidad funcional, ya que presupone que la diversidad y lo diferente es dañino. Este grupo lo nutren las series: El chiringuito de Pepe (Telecinco, 2014-2016), en la que el personaje con diversidad funcional es Vicente, el cual se presenta como un personaje bueno, simpático y algo ingenuo. El actor que encarna a este personaje es Juan Manuel Montilla, más conocido como 'El Langui', cantante del grupo madrileño La Excepción.

En la misma tónica que el anterior caso se sitúa Ella es tu padre (Telecinco, 2017-2018), donde el personaje con diversidad funcional en este caso es Yago, el batería del grupo de Sergio, que es ciego. Es un personaje secundario carente de objetivos narrativos propios más allá de ayudar al protagonista y que se muestra como una persona poco autónoma en la realización de actividades cotidianas, como el desplazamiento de un lugar a otro o la interacción con otros personajes. Por otro lado, las referencias a la diversidad funcional de este personaje son constantes, muchas de las cuales son hechas por el propio Yago.

VINI: Lo que se ha caído es un cacho de techo, ciego de los cojones.

CLOE: ¡Eres ciego!

YAGo: Solo en un noventa y un casi nada por ciento. Algún día conduciré solo de oído.

El siguiente caso incluido en este grupo es el de El Continental (TVE, 2018), donde el personaje con diversidad funcional es Perico, un tartamudo que trabaja de camarero en el bar El Continental. Por lo tanto, su relevancia narrativa se reduce a ser un personaje secundario. Alfonso, el dueño del local, encarga a Perico que cuide de su hija y que se la lleve a París con él para asegurarse de que está lejos de Ricardo, antihéroe de la serie. Sin embargo, falla en la misión y no solo se quedan en El Continental, sino que Andrea, hija de Alfonso, comienza a sentirse 


\section{DISERTACIONES}

AVANCES

atraída por Ricardo. Así, Perico se muestra como un complemento de los personajes principales, un ayudante que en determinados casos sirve como objeto de mofa y burla. No obstante, al ser retratado como un personaje amable y afable, consigue el cariño de todos, incluso de Ricardo.

Por último, en este grupo se localiza la serie Cuéntame cómo pasó (TVE, 2001-actualidad). En esta ocasión, el personaje con diversidad funcional es Gonzalo, un chico mudo de 13 años que se da un beso con María, la hija pequeña de los Alcántara. En esta ocasión sí que se hace referencia a la diversidad funcional del personaje de manera directa, indicando su tipología. Está representado como un chico con mucha confianza en sí mismo, no se avergüenza de ser mudo y en la narración se puede observar una comparativa con María, que se avergüenza de sus recién adquiridas gafas. Sí que es cierto que, aunque no se hace una ridiculización directa del personaje por el hecho de ser mudo, hay una diferencia en la aceptación social entre las gafas de María y el hecho de que Gonzalo sea mudo, ya que mientras que María tiene un apoyo por parte de su círculo familiar y de amistades, Gonzalo es acusado de ser tonto o no entender a las personas por no poder contestar.

MARIA: ¿Tú qué miras? Llevo gafas, ¿qué pasa? Dice mi amiga Gala que me dan un aire intelectual. ¿Es verdad que de pequeño te dieron un susto y ya nunca has vuelto a hablar? Te he visto patinar en la calle con ese Sancheski tan chulo que tienes. ¿Que quieres mis gafas? Pero no me las rompas, ¿eh? ¡Que te llevas mis gafas! ¡Que valen 5000 pesetas!

Al final del relato, María se acaba dando un beso con Gonzalo y la abuela, Herminia, muy enfadada va a hablar con el abuelo de este.

HERMINIA: ¡Eh, tú, chaval! No te escondas. Eladio, dile al pinta de tu nieto que venga aquí.

ELADIO: ¿Qué ha hecho?

HERMINIA: Que te lo diga él.

ELADIO: No puede hablar, es mudo.

HERMINIA: ¿Cómo que es mudo?

ELADIO: ¡Gonzalo, ven para acá! ¡Vamos! ¡Gonzalo, ven para acá! ¿Qué has hecho?

HERMINIA: ¡Habla!

ELADIO: No puede hablar, es mudo.

HERMINIA: ¿Cómo que es mudo?

ELADIO: Pues eso, coño. Que es mudo.

HERMINIA: ¿Y sordo también?

ELADIO: No, solo mudo.

\section{Conclusiones}

Como conclusión principal y una vez realizada la descripción de los personajes que nutren la muestra realizada y analizado el papel que cumplen en ella, se puede afirmar que los personajes con diversidad funcional son en su mayoría personajes que ocupan papeles secundarios o de background, por lo que funcionan más como apoyo al personaje protagonista que como personajes que luchan por alcanzar un objetivo propio. Por otro lado, la autonomía 


\section{DISERTACIONES}

AVANCES

Comunicación y controversias sociocientíficas en salud y medio ambiente

ISSN: 1856-9536

Doi: https://doi.org/10.12804/revistas.urosario.edu.co/disertaciones/a.1012.1

Volumen 14, Número 2 / Julio-diciembre 2021

Versión PDF para imprimir desde

http://revistas.urosario.edu.co/index.php/disertaciones

que estos muestran en la narración es prácticamente inexistente, pues en su mayoría dependen de otros personajes para poder llevar a cabo acciones cotidianas e, incluso, llega a quedar anulada su capacidad de decisión sobre sus propios actos. Esta dependencia se ve reflejada en series como Fugitiva (TVE, 2018), El incidente (Antena 3, 2017) o Mar de plástico (Antena 3, 2015-2016), donde los personajes con diversidad funcional se muestran completamente dependientes de otras personas.

Otro punto importante es que siempre que estos personajes están involucrados en una relación amorosa o se plantea que puedan desarrollar este tipo de relaciones enseguida se anula esa idea. Este concepto acaba mutando en que los personajes normativos terminen estableciendo el vínculo afectivo entre ellos, separando al personaje con diversidad funcional al plano de amistad y tratándose siempre de una relación completamente heteronormativa, como en el caso de El Continental (tve, 2018) o en El chiringuito de Pepe (Telecinco, 2014-2016). La excepción es Cuéntame cómo pasó (TVE, 2001-actualidad), donde Gonzalo es el personaje que da el paso de besar a María, aunque es cierto que en ningún momento del capítulo se indica que la relación pueda seguir hacia algo más emocional o se vaya a quedar en un plano más anecdótico.

Como añadido al presente análisis, hay que señalar el hecho de que no existe en la muestra representación de mujeres con diversidad funcional. Esto es notable, dado que en el estudio "Representación de género en las series generalistas de televisión españolas emitidas en prime time (2017-2018)" (De Caso et al., 2020), el cual comparte método y una parte de la muestra usada en el análisis previo a este estudio, ya se hacía notable la infrarrepresentación de mujeres en el marco de la ficción nacional, donde el 40,7\% de la muestra correspondía a personajes femeninos. Es decir, de los 514 personajes analizados en dicho estudio, 209 correspondían a personajes femeninos y solamente un $0,2 \%(n=1)$ a personajes no binarios. Por lo tanto, la diversidad funcional sería representada en la muestra por personajes leídos como hombres heterosexuales (pues no existe en la muestra ningún ejemplo de otra sexualidad), la subrepresentación en este caso sería doble y, por ende, la invisibilización también.

Por otro lado, el paternalismo mostrado con relación a determinados personajes como Sergio en Mar de plástico (Antena 3, 2015-2016) o con Rubén en Fugitiva (TVE, 2018) mantienen la concepción de que pertenecer al colectivo de hombres y mujeres con diversidad funcional significa una incapacidad para tomar decisiones por uno mismo. Es decir, aparte de perpetuar la perspectiva de que diversidad funcional es lo mismo que enfermedad, se añade la concepción de que las personas con diversidad funcional no pueden tomar decisiones por sí mismas debido precisamente a esa supuesta 'enfermedad'. Esta vía de representación, en conjunto con la visión infantilizada de personajes como Vicente en El chiringuito de Pepe (Telecinco, 2014 -2016) o Perico en la serie El Continental (TVE, 2018), hace que el discurso de autonomía para estos personajes no se vea desarrollado y se perpetúe la infantilización hacia ellos.

No obstante, en los casos de Cuéntame cómo pasó (TVE, 2001-actualidad) y Ella es tu padre (Telecinco, 20172018), los personajes que aparecen en la narración aluden abiertamente a su diversidad funcional, no la esconden y su círculo habla sobre ello de manera normativa, incluso haciendo bromas. Este modelo de representación estaría muy relacionado con el actual, propio de narraciones como Campeones (Javier Fesser, 2018), en el que, pese a que el personaje principal sigue sin pertenecer al colectivo de personas con diversidad funcional, esta se muestra de una manera positiva, sin que sea un impedimento para que los personajes puedan realizar actividades cotidianas. Además, en esta película ningún actor ficciona la diversidad funcional que aparece en pantalla, hecho que se comparte en la muestra en la que se ha basado este artículo, ya que también existe el caso en que 


\section{DISERTACIONES}

AVANCES

Comunicación y controversias sociocientíficas en salud y medio ambiente

ISSN: 1856-9536

Doi: https://doi.org/10.12804/revistas.urosario.edu.co/disertaciones/a.1012.1

Volumen 14, Número 2 / Julio-diciembre 2021

Versión PDF para imprimir desde

http://revistas.urosario.edu.co/index.php/disertaciones

una persona con diversidad funcional es el actor que encarna y representa al colectivo. Se trata de Juan Manuel Montilla, que aparece como Vicente en El chiringuito de Pepe (Telecinco, 2014-2016) y como Ramón Carreño en Cuerpo de Élite (Antena 3, 2018). Sin embargo, la diferencia radica en que, además de que es el mismo actor en ambas ocasiones, 'El Langui' (como se dio a conocer), ya era famoso por su actividad musical antes de comenzar su carrera como actor. Por lo tanto, habría que plantear si la industria de la ficción nacional es realmente inclusiva en términos de inserción laboral. Siguiendo con el ejemplo de Juan Manuel Montilla encarnando a Ramón Carreño en la serie Cuerpo de Élite (Antena 3, 2018), se ha considerado importante añadir que el personaje que representa es un político que viene de una tradición de sindicalismo estudiantil y curtido durante la época del 15-M, lo que no deja de trasladar al espectador a la figura de Pablo Echenique, político que también se traslada en una silla de ruedas y que políticamente tiene la misma inclinación que el personaje referido. Es decir, no solo se propone como regla general que la diversidad funcional sea representada desde una perspectiva paternalista o de enfermedad, sino que a la hora de proponer discursos alternativos se recurre a figuras que ya son reconocidas socialmente. Por un lado, el actor ya es famoso por su carrera musical y, por otro lado, el personaje ya es reconocido por la similitud al ya nombrado Pablo Echenique.

Otro de los modelos y roles que se pueden observar en el modo de representar la diversidad funcional en las series de ficción españolas durante el periodo indicado es el de recurrir a la representación de la diversidad como enfermedad, casos como el de José en El incidente (Antena 3, 2017), donde todas las personas con diversidad funcional aparecidas en la ficción son representadas como enfermas y, en efecto, la narración hace hincapié en que el hecho de su recuperación es algo positivo, dado que se puede superar la enfermedad que supone la diversidad funcional manifestada. En el caso de Sergio en Mar de plástico (Antena 3, 2015-2016), en el que la diversidad funcional está representada como parte de la locura que lleva al personaje a cometer crímenes es parte de la representación que se hace de la diversidad funcional. Sin embargo, según el modelo de vida independiente que se intenta seguir en este artículo, la diversidad funcional no siempre tiene que estar relacionada con la enfermedad, pues existen casos en que la pertenencia a este colectivo puede darse sin necesidad de manifestar una enfermedad y viceversa (Palacios \& Romañach, 2006). Por ejemplo, Gonzalo en Cuéntame cómo pasó (Tve, 2001-actualidad) es un personaje mudo, pero que no reniega de realizar actividades propias de su edad, ni muestra una actitud sumisa, poca confianza en sí mismo, ni tampoco muestras de enfermedad por el hecho de ser mudo. Así mismo, el caso de María Alcántara en Cuéntame cómo pasó (TVE, 2001-actualidad) es llamativo, ya que, aunque para la mayoría de los espectadores el llevar gafas es algo común, ella no se siente cómoda porque se ve diferente respecto a la mayoría, no porque no pueda ver con normalidad, sino por las gafas que marcan una diferencia con el resto.

Como conclusión general y siguiendo la concepción del modelo de vida independiente (Palacios \& Romañach, 2006), las narraciones de ficción españolas que se recogen en esta muestra alimentarían la concepción de la diversidad funcional como algo negativo, que bien hay que esconder o que bien hay que resaltar como concepto de una visión paternalista. Se mantiene la representación de los tres modelos previos al modelo de vida independiente, representando y asociando la diversidad funcional a la enfermedad, a la dependencia y, en varias ocasiones, a la anulación de la voluntad del personaje con diversidad funcional, infantilizándolo y perpetuando una imagen paternalista de este. A la luz de la escasez y estereotipación de los relatos parece necesario que se creen modelos alternativos de comunicación para los colectivos sociales minoritarios que han estado relegados a un segundo 


\section{DISERTACIONES}

AVANCES

plano. Es fundamental que su representación se haga desde otra visión, otorgando una perspectiva de independencia y autonomía. No obstante, las nuevas narraciones audiovisuales españolas de plataforma de video bajo demanda con presencia de personajes con diversidad funcional parecen apuntar a un cambio de paradigma. Ejemplos como el de Laura en Merlí: Sapere Aude (Movistar+, 2019) o Gari en Vida perfecta (Movistar+, 2019) sí que muestran un avance en cuanto al modelo de representación que en ellas aparecen, dado que se trata de personajes con un importante peso narrativo, fuertes e independientes, que no consideran su diversidad como algo negativo a lo que tienen que superponerse. Además, se introducen temas tabúes como la sexualidad en las personas con diversidad funcional. Con respecto a futuras líneas de investigación, se propone ampliar el aspecto de la muestra hacia una revisión de las plataformas online que tienen contenido audiovisual, como Netflix, ATREsplayer, YouTube o Playz, pues cada vez son más populares entre los espectadores, sobre todo en las franjas de edad más jóvenes.

\section{Referencias}

1. Alegre de la Rosa, O. M. (2002). La discapacidad en el cine: Propuestas para la acción educativa. Comunicar, 18, 130-136. https://doi.org/10.3916/C18-2002-25

2. Areces Gutiérrez, R. (2017). La discapacidad en el cine [tesis doctoral, Universidad Complutense de Madrid, España]. https://eprints.ucm.es/42223/

3. Belmonte, J., \& Guillamón, S. (2008). Cómo co-educar la mirada contra los estereotipos de género en Tv. Comunicar, 31(16), 115-120. https://doi.org/10.3916/c31-2008-01-014

4. Cebrián Herreros, M. (2010). Percepción de las personas con discapacidad por los profesionales de los medios de comunicación. Fundación oncE. http://sid.usal.es/idocs/F8/FDO24796/percepcion_imagen.pdf

5. Cohen, J. (2001). Defining identification: A theoretical look at the identification of audiences with media characters. Mass Communication and Society, 4(3), 245-264. https://tinyurl.com/yyqch2ax

6. De Caso Bausela, E., González de Garay, B., \& Marcos Ramos, M. (2020). Representación de género en las series generalistas de televisión españolas emitidas en prime time (2017-2018). El Profesional de la Información, 29(2). https://doi.org/10.3145/epi.2020.mar.08

7. Del Río, P., \& Álvarez, A. (2004). Informe Pigmalión. Fundación Infancia y Aprendizaje-Centro Nacional de Información y Comunicación Educativa. http://ares.cnice.mec.es/informes/03/documentos/indice.htm

8. España, Ministerio de Sanidad, Servicios Sociales e Igualdad. Real Decreto Legislativo 1/2013 del 29 de noviembre. Texto refundido de la Ley General de Derechos de las Personas con Discapacidad y de su Inclusión Social. Boletín Oficial del Estado 289, 3 de diciembre de 2013, pp. 95635-95673. https://www.boe.es/ eli/es/rdlg/2013/11/29/1

9. Ferrés i Prats, J. (2003). Educación en medios y competencia emocional. Revista Iberoamericana de Educación, 32, 49-69. https://doi.org/10.35362/rie320920

10. Ferrés i Prats, J., \& Masanet Jordà, M. J. (2017). La eficacia comunicativa en la comunicación: Potenciando las emociones y el relato. Comunicar, 52, 51-60. https://doi.org/10.3916/C52-2017-05

11. Fesser, J. (Director). (2018). Campeones [Película]. Morena Films.

12. Galán Fajardo, E. (2006). Personajes, estereotipos y representaciones sociales: Una propuesta de estudio y análisis dela ficción televisiva.Eco-Pós, 9(1),55-81. https://e-archivo.uc3m.es/handle/10016/9475\#preview 


\section{DISERTACIONES}

AVANCES

Comunicación y controversias sociocientificas en salud y medio ambiente

ISSN: 1856-9536

Doi: https://doi.org/10.12804/revistas.urosario.edu.co/disertaciones/a.10121

Volumen 14, Número 2 / Julio-diciembre 2021

Versión PDF para imprimir desde

http://revistas.urosario.edu.co/index.php/disertaciones

13. Herrero Jiménez, B., \& Tovar, M. (2011). Cine y discapacidad: La construcción de la identidad del otro. Un caso de estudio: La vida secreta de las palabras (Isabel Coixet, 2005). En I Jornadas Universitarias de Comunicación y Personas con Discapacidad (3351), Universidad de Sevilla, Sevilla. https://tinyurl.com/y6qt4l86

14. Igartua Perosanz, J. J. (2006). Métodos cuantitativos de investigación en comunicación. Bosch.

15. Igartua Perosanz, J. J., \& Humanes, M. L. (2004). Teoría e investigación en comunicación social. Madrid: Síntesis.

16. Igartua Perosanz, J. J., \& Marcos Ramos, M. (2014). Creación de personajes para la ficción televisiva: Influencia del tipo de personaje y del contexto de la acción narrativa. En I. Fernández-Astorbiza (Ed.), Espacios de la comunicación. Actas del IV Congreso Internacional de la AE-IC (pp. 1101-1115). AE-IC-Universidad del País Vasco. https://tinyurl.com/y297rto2

17. Instituto Nacional de Estadística (INE). (2019). Encuesta sobre equipamiento y uso de tecnologías de información y comunicación en los hogares año 2019. https://tinyurl.com/ybhmxwaf

18. Jiménez, A., \& Huete, A. (2017). Informe Olivenza 2017, sobre la situación general de la discapacidad en España. Observatorio Estatal de la Discapacidad. https://www.cedd.net/es/documentacion/catalogo/ Record/533382

19. Lacalle, C., \& Gómez Morales, B. (2018). Educar entreteniendo: Los problemas de salud de los personajes femeninos en la ficción tv española. Signo y Pensamiento, 37(73). https://doi.org/10.11144/Javeriana. syp37-73.eeps

20. Marcos Ramos, M., González de Garay, B., \& Portillo Delgado, C. (2019). La representación de la inmigración en la ficción serial española contemporánea de prime time. Revista Latina de Comunicación Social, 74, 285-307. https://doi.org/10.4185/RLCS-2019-1331

21. Mejía Zapata, S. I. (2020). Diversidad funcional, emergente de un enfoque de derechos y autodeterminación. Tempus Psicológico, 3(2), 155-171. https://doi.org/10.30554/tempuspsi.3.2.3695.2020

22. Miranda Cáceres, R. (2003). Nuevos modelos de producción audiovisual. Revista Latina de Comunicación Social, 54. http://www.ull.es/publicaciones/latina/200353miranda.htm

23. Monjas Casares, M. I., \& Arranz Moro, F. (2010). El cine como recurso para el conocimiento de las personas con discapacidad: Veinticinco películas de la última década. Revista de Medicina y Cine, 6(2). https://tinyurl. com/y6n6xdg4

24. Palacios, A., \& Romañach, J. (2006). El modelo de la diversidad: La bioética y los derechos humanos como herramientas para alcanzar la plena dignidad en la diversidad funcional. Ediciones Diversitas-AlEs. https:// e-archivo.uc3m.es/handle/10016/9899\#preview

25. Palacios, A., \& Romañach, J. (2008). El modelo de la diversidad: Una nueva visión de la bioética desde la perspectiva de las personas con diversidad funcional (discapacidad). Intersticios: Revista Sociológica de Pensamiento Crítico, 2(2), 37-47. http://www.intersticios.es/article/view/2712

26. Palenzuela Zanca, J., Marcos Ramos, M., \& González de Garay Domínguez, B. (2019). Representación de la diversidad funcional en series contemporáneas españolas de 'prime time'. index.comunicación, 9(3), 165-183. https://tinyurl.com/y6yeyo5h

27. Peñamarín, C. (2001). Ficción televisiva y pensamiento narrativo. Universidad Carlos III de Madrid. https:// tinyurl.com/y55c5x45 
28. Pérez Tornero, J. M. (2008). La sociedad multipantallas: Retos para la alfabetización mediática. Comunicar, 31, 15-25. https://doi.org/10.3916/c31-2008-01-002

29. Romañach, J., \& Lobato, M. (26 de julio de 2009). Diversidad funcional, nuevo término para la lucha por la dignidad en la diversidad del ser humano. Foro de Vida Independiente y Divertad. https://tinyurl.com/ y5hk7e5x

30. Senent, M. (2012). El dolor de la diferencia: Cine y diversidad funcional. Dossiers Feministes, 16, 79-92. https://dialnet.unirioja.es/servlet/articulo? codigo $=4235761$

31. Tabernero, C., Sánchez Navarro, J., Aranda, D., \& Tubella, I. (2009). Transformación de prácticas comunicativas en contextos de multiplicación de pantallas. Metamorfosis de los Formatos en el Ámbito Publicitario, (2), 1011-1020.

32. Toboso Martín, M. (2013). De los discursos actuales sobre la discapacidad en España. Política y Sociedad, 50(2), 681-706. https://doi.org/10.5209/rev_POSO.2013.v50.n2.39007

33. Ugalde, L., Medrano, M. C., \& Aierbe, A. (2012). Identificación con personajes cinematográficos discapacitados y valores percibidos: Una investigación experimental con universitarios. zER: Revista de Estudios de Comunicación, 17(32). https://tinyurl.com/y523yw2n

34. Van Dijk, T. A. (2005). Ideología y análisis del discurso. Utopía y Praxis Latinoamericana, 10(29), 9-3. https:// tinyurl.com/kl562dd

35. Vázquez Ferreira, M. Á. (2008). Una aproximación sociológica a la discapacidad desde el modelo social: Apuntes caracteriológicos. Revista Española de Investigaciones Sociológicas (REIS), 124(1), 141-174. http://www.reis.cis.es/REIS/PDF/REIS_124_051222873458779.pdf 